\title{
Sur la mesure de la température de l'oreille externe.
}

\author{
Par \\ Watari Matsumoto. \\ (De la clinique de la laryngologie d'otologie et de rhinologie à l'Université Impériale \\ de Kyoto. Directeur: Prof. T. Hoshrno.). \\ et \\ Hisawo Nakagawa.
}

(De la clinique des maladies internes à l'Université Impériale de Kyoto.

Directeur: Prof. S. Mashumo.)

Recémment il y a beaucoup d'étudiants qui travaillent pour savoir la fonction de l'échange nutritif en prenant la température des organs et des tissus, mais cependant, nous n'en savons pas qui travaillent sur l'organe de l'audition. Quand nous expérimentons sur la température en nous servant du galvanomètre pour faire l'étude expérimentale de la voie de conduction du chaleur à l'égard de l'apparition du nystagmus calorique, nous prenons la température du conduit auditif externe et celui du sous-cutané: c'est pour expliquer notre expérience que nous faisons le rapport ci-après.

L'apparatus de l'expérience.

Nous choisissons des lapins domestiques normaux et nous prenons la température de l'anus et du conduit auditif externe où nous faisons entrer directment le thermomètre et nous prenons la température du muscle du dos à une petite blessure où se met lo thermomètre prenant garde autant que possible que la hemorrhagie ne se produise. La température du conduit auditif externe est calculée par le tableau déjà fait au moyen du mouvement du galvanomètre en utilisant le courant thermo-électrique. C'est-à -dire nous employons l'apparatus suivant.

Nous attachons un fil du cuivre no. 40 de grandeur à un fil de constantan de même grandeur (Voyez le tableau) et nous faisons la thermo-couple en soudant réciproquement les extrémités des deux fils en métal par de l'argent. Une thermocouple est fixée à un bain où se soutient la température constante de $37^{\circ} \mathrm{C}$, l'autre thermo-couple est percé dans la canule en manière de traverser le troicart dans le 
tissu qu'on va observer. Le courant thermo-électrique est lu par le mouvement du galvanomètre ainsi que nous pourons prendre la température. Nous examinons

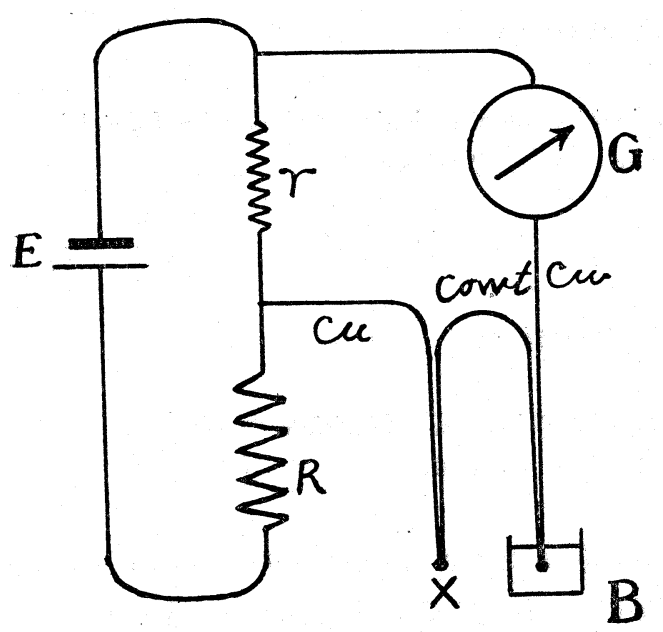

G.........Galvanomètre

R.........La petite resistance

R........La grande resistauce

E.........La pile électrique

Cu.........Le fil de cuivre

Const.........Le fil de constantan

B.......... Le bain de $37,0^{\circ} \mathrm{C}$

$\mathrm{X}$........... Le thermo couple examiné

plusieurs fois combien de centimètre se meut le galvanomètre pour s' élever et se baisser par degrés de $32^{\circ}$ à $42^{\circ} \mathrm{C}$ : voici le résultat:-

\begin{tabular}{|r|c|c|c|c|c|c|c|c|c|c|c|}
\hline Fois & $32^{\circ}$ & $33^{\circ}$ & $34^{\circ}$ & $35^{\circ}$ & $36^{\circ}$ & $37^{\circ}$ & $.38^{\circ}$ & $39^{\circ}$ & $40^{\circ}$ & $41^{\circ}$ & $42^{\circ}$ \\
\hline 1 & 5,0 & 5,0 & 5,8 & 5,0 & 5,0 & 0 & 5,0 & 4,8 & 4,8 & 5,2 & 5.0 \\
2 & 4,4 & 4,0 & 5,1 & 4,8 & 4,7 & 0 & 4,3 & 4,9 & 4,7 & 5,3 & 4,8 \\
3 & 4,8 & 3,8 & 6,0 & 4,8 & 5,3 & 0 & 4,9 & 4,5 & 5,4 & 5,0 & 4,7 \\
4 & 4,3 & 5,5 & 5,0 & 4,5 & 5,0 & 0 & 5,0 & 5,1 & 5,8 & 5,1 & 5,1 \\
\hline \multirow{2}{*}{ Moven } & 4,6 & 4,9 & 5,5 & 4,9 & 5,0 & 0 & 4,8 & 4,8 & 5,2 & 5,1 & 5,0 \\
\hline
\end{tabular}

Dans cette expérience, le zéro du galvanomètre represente $37^{\circ} \mathrm{C}$.

Les mouvements de l'aiguille sont 4,98 centimètre en moyenne par degré et nous pouvons faire le tableau suivant à l'aide du résultat déjà écrit. 


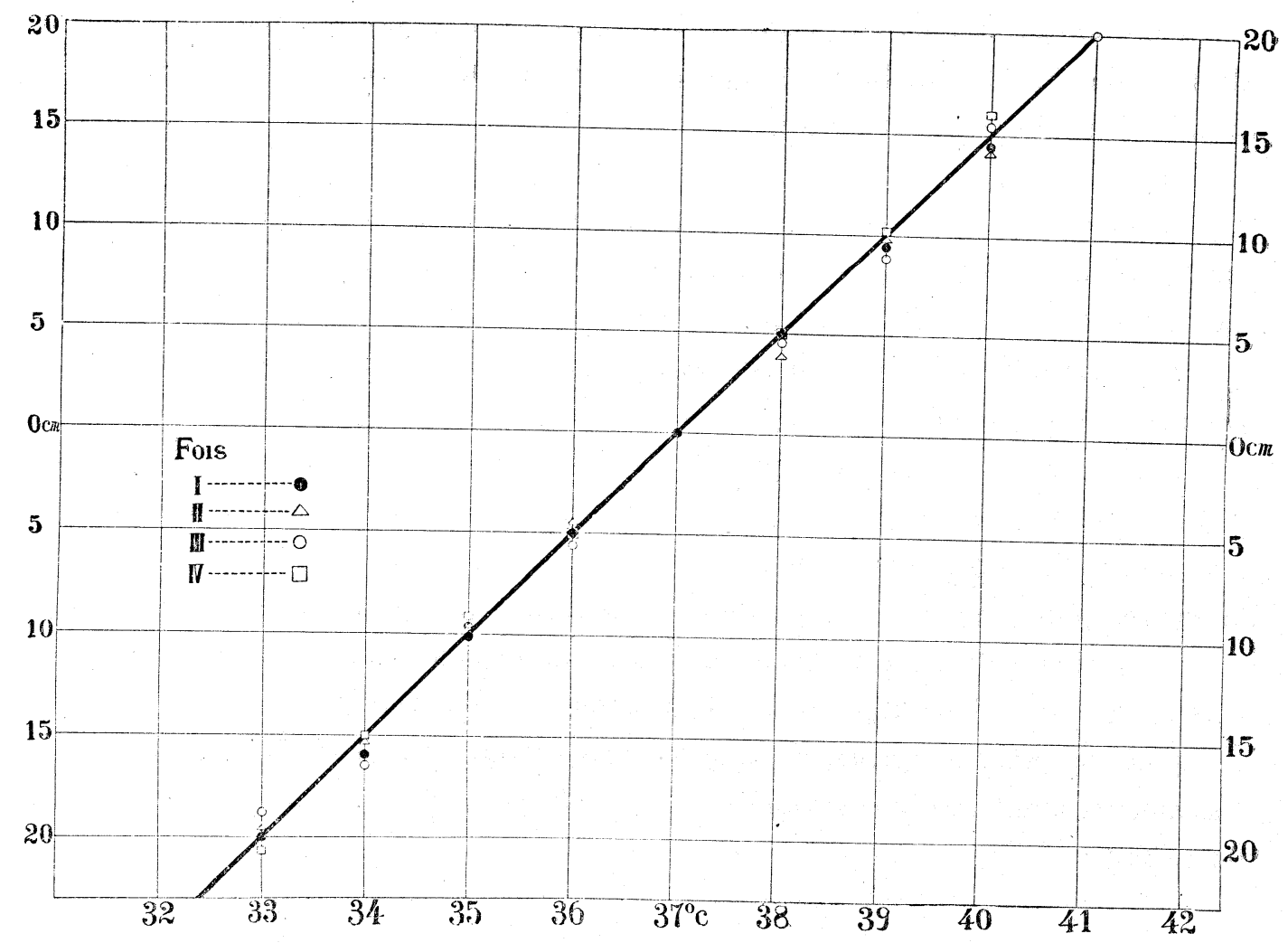

Le galvanomètre employé pour cet but mesure jusqu'à $10^{-7}$ milliampère et tout est fait dans la chambre à la température de $10^{\circ}$ à $17^{\circ} \mathrm{C}$.

Le résultat des expériences

La tempèrature prise du conduit auditif externe et du sous-cutané des lapins domestiques est comme suite.

\begin{tabular}{|c|c|c|c|c|c|}
\hline $\begin{array}{c}\text { Le numero des } \\
\text { lapins } \\
\text { domestiques }\end{array}$ & $\begin{array}{c}\text { La température } \\
\text { de la chambre }\end{array}$ & $\begin{array}{c}\text { La températnre } \\
\text { de l'anus }\end{array}$ & $\begin{array}{c}\text { La température } \\
\text { du muscle } \\
\text { du dos }\end{array}$ & $\begin{array}{c}\text { La température, } \\
\text { de la sous-cutané } \\
\text { du conduit } \\
\text { auditif externe }\end{array}$ & $\begin{array}{c}\text { La température } \\
\text { du conduit } \\
\text { auditif externe }\end{array}$ \\
\hline 1 & $10^{\circ} \mathrm{C}$ & $38,3^{\circ} \mathrm{C}$ & $38,1^{\circ} \mathrm{C}$ & $37,6^{\circ} \mathrm{C}$ & $379^{\circ} \mathrm{C}$ \\
2 & 11 & 38,0 & 37,9 & 37,4 & 37,1 \\
3 & 11 & 37,7 & 36,6 & 37,5 & 36,0 \\
4 & 11 & 37,6 & 37,5 & 37,2 & 36,3 \\
5 & 12 & 37,3 & 37,8 & 36,7 & 35,2 \\
6 & 12 & 37,3 & 37,8 & 36,7 & 37,2 \\
8 & 11 & 38,7 & 38,4 & 37,2 & 37,0 \\
9 & 12 & 38,5 & 38,0 & 37,4 & 37,3 \\
10 & 17 & 37,8 & 37,4 & 37,3 & 37,4 \\
\hline Moyen & 11,9 & 37,9 & 37,62 & 37,21 & 36,35 \\
\hline
\end{tabular}


Les température en moyenne de dix lapins domestiques qui sont mis dans la chambre de $11,9^{\circ} \mathrm{C}$ sont $37,9^{\circ}$ à l'anus, $37,63^{\circ}$ au muscle du dos, $37,21^{\circ}$ au souscutané du conduit auditif externe.

La considération.

La température du conduit auditif externe du lapin domestique est prise à la conque (c'est-à-dire à la partie attacheé du pavillon de l'oreille), comme la situation du conduit auditif externe du lapins est different de celle de l'homme. Nous pensons que la température de cette partie est beaucoup plus basse que celle du corps de l'animal, mais la différence n'est que $1.5^{\circ}$ environ.

On enumére comme la cause de la congélation du pavillon de l'oreille de l'homme, la disposition constitutionelle, l'exposition de local, l'insuffisance du tissu sous-cutané. l'état de la circulation du sang, non-mouvement du pavillon de l'oreille, la surface plus grande en comparaison de la capacité de pavillon de l'oreille etc.

La différence de la température n'est pas remarquable, parce que le pavillon de l'oreille du lapin domestique se meut activement et est couvert de cheveux. Alors on peut facilement conjecturer que la température du pavillon de l'oreille de l'homme est plus basse en comparaison de celle du corps de l'homme en considération de ce fait. 